\title{
Note on antisymmetric spin-tensors
}

\author{
Yu. M. Zinoviev* \\ Institute for High Energy Physics \\ Protvino, Moscow Region, 142280, Russia
}

\begin{abstract}
It was known for a long time that in $d=4$ dimensions it is impossible to construct the Lagrangian for antisymmetric second rank spin-tensor that will be invariant under the gauge transformations with unconstrained spin-vector parameter. But recently a paper [5] appeared where gauge invariant Lagrangians for antisymmetric spin-tensors of arbitrary rank $n$ in $d>2 n$ were constructed using powerful BRST approach. To clarify apparent contradiction, in this note we carry a direct independent analysis of the most general first order Lagrangian for the massless antisymmetric spin-tensor of second rank. Our analysis shows that gauge invariant Lagrangian does exist but in $d \geq 5$ dimensions only, while in $d=4$ this Lagrangian becomes identically zero. As a byproduct, we obtain a very simple and convenient form of this massless Lagrangian that makes deformation to $A d S$ space and/or massive case a simple task as we explicitly show here. Moreover, this simple form admits natural and straightforward generalization on the case of massive antisymmetric spin-tensors of rank $n$ for $d>2 n$.
\end{abstract}

\footnotetext{
${ }^{*}$ E-mail address: Yurii.Zinoviev@ihep.ru
} 


\section{Introduction}

It was known for a long time that in $d=4$ dimensions it is impossible to construct the Lagrangian for antisymmetric second rank spin-tensor that will be invariant under the gauge transformations with unconstrained spin-vector parameter [1, 2, 3] (see also more recent paper [4]). But recently a paper [5] appeared where gauge invariant Lagrangians for antisymmetric spin-tensors of arbitrary rank $n$ in $d>2 n$ were constructed using powerful BRST approach [6, 7, 8, 9, 10, 11, 12]. The aim of this short note is to clarify an apparent contradiction between these new and previous results.

The paper structured as follows. In Section 1, as a warming up exercise, we consider simplest and very well known case of spin-vector field $\Psi_{\mu}$. Such field can be considered as a simplest example of completely antisymmetric spin-tensors and indeed this case gives some useful hints. Then in Section 2 we consider the most general first order Lagrangian for massless antisymmetric spin-tensor $\Psi_{\mu \nu}$ in flat Minkowski space and show that the Lagrangian invariant under the gauge transformations $\delta \Psi_{\mu \nu}=\partial_{\mu} \xi_{\nu}-\partial_{\nu} \xi_{\mu}$ with unconstrained spinvector parameter $\xi_{\mu}$ does exist, but in $d \geq 5$ dimensions only, while it becomes identically zero in $d=4$. As a byproduct we obtain a very simple and convenient form of this gauge invariant Lagrangian that makes deformation into $A d S$ space a simple task as we explicitly show here.

In Section 3 we consider gauge invariant description of massive antisymmetric spin-tensor using a minimalistic approach as in [13, 14, 15, 16, 17, 18. Due to reducibility of gauge transformations it turns out enough to have two fields - spin-tensor $\Psi_{\mu \nu}$ and spin-vector $\Phi_{\mu}$ only. At last, in Section 4 we show that these results admit a natural and straightforward generalization on the case of spin-tensors with arbitrary rank $n$ in $d \geq 2 n+1$. Again, due to reducibility of gauge transformations, to obtain gauge invariant description of massive field it is enough to introduce two spin-tensors with rank $n$ and $n-1$ only.

\section{Warming up exercise}

As a warming up exercise let us take an example of spin-vector $\Psi_{\mu}$ (any way it can be considered as a simplest case of completely antisymmetric spin-tensors). The most general first order Lagrangian for massless field in flat Minkowski space can be written as follows:

$$
\frac{1}{i} \mathcal{L}_{0}=\bar{\Psi}^{\mu} \hat{\partial} \Psi_{\mu}+a_{1}((\bar{\Psi} \partial)(\gamma \Psi)+(\bar{\Psi} \gamma)(\partial \Psi))+a_{2}(\bar{\Psi} \gamma) \hat{\partial}(\gamma \Psi)
$$

Now if we require that this Lagrangian be invariant under the gauge transformations $\delta \Psi_{\mu}=$ $\partial_{\mu} \xi$ with spinor parameter, we immediately obtain:

$$
\partial^{\mu} \frac{\delta \mathcal{L}_{0}}{\delta \bar{\Psi}^{\mu}}=0 \quad \Rightarrow \quad a_{1}=-1, \quad a_{2}=1
$$

First signal that something happens then $d=2$ comes from the contraction of equation with $\gamma$-matrix:

$$
\gamma^{\mu} \frac{\delta \mathcal{L}_{0}}{\delta \bar{\Psi}^{\mu}}=i(d-2)[\hat{\partial}(\gamma \Psi)-(\partial \Psi)]
$$


Noting that in $d=2$ the last term in the Lagrangian containing three $\gamma$-matrices is not independent, let us rewrite the Lagrangian in terms of completely antisymmetric products of $\gamma$-s. Using the following relation:

$$
\gamma^{\mu} \gamma^{\nu} \gamma^{\alpha}=g^{\mu \nu} \gamma^{\alpha}-g^{\mu \alpha} \gamma^{\nu}+g^{\nu \alpha} \gamma^{\mu}+\Gamma^{\mu \nu \alpha}
$$

where $\Gamma^{\mu \nu \alpha}=\gamma^{[\mu} \gamma^{\nu} \gamma^{\alpha]}$, the most general Lagrangian can be written as follows:

$$
\begin{aligned}
\frac{1}{i} \mathcal{L}_{0} & =\left(1-a_{2}\right) \bar{\Psi}^{\mu} \hat{\partial} \Psi_{\mu}+\left(a_{1}+a_{2}\right)((\bar{\Psi} \partial)(\gamma \Psi)+(\bar{\Psi} \gamma)(\partial \Psi))+a_{2} \bar{\Psi}_{\mu} \Gamma^{\mu \nu \alpha} \partial_{\nu} \Psi_{\alpha}= \\
& =\bar{\Psi}_{\mu} \Gamma^{\mu \nu \alpha} \partial_{\nu} \Psi_{\alpha}
\end{aligned}
$$

where the last line corresponds to the same values of parameters when the Lagrangian is gauge invariant. In this form gauge invariance of the Lagrangian is evident as well as the fact that it is identically zero in $d=2$.

\section{Massless case}

In this Section we consider massless antisymmetric spin-tensor $\Psi_{\mu \nu}$. The most general first order Lagrangian can be written in the following form:

$$
\begin{aligned}
\frac{1}{i} \mathcal{L}_{0}= & \bar{\Psi}^{\mu \nu} \hat{\partial} \Psi_{\mu \nu}+a_{1}\left((\bar{\Psi} \partial)^{\mu}(\gamma \Psi)_{\mu}+(\bar{\Psi} \gamma)^{\mu}(\partial \Psi)_{\mu}\right)+a_{2}(\bar{\Psi} \gamma)^{\mu} \hat{\partial}(\gamma \Psi)_{\mu}+ \\
& +a_{3}((\bar{\Psi} \partial \gamma)(\gamma \gamma \Psi)-(\bar{\Psi} \gamma \gamma)(\partial \gamma \Psi))+a_{4}(\bar{\Psi} \gamma \gamma) \hat{\partial}(\gamma \gamma \Psi)
\end{aligned}
$$

where $(\gamma \Psi)_{\nu}=\gamma^{\mu} \Psi_{\mu \nu},(\bar{\Psi} \gamma)^{\nu}=\bar{\Psi}^{\mu \nu} \gamma_{\mu}$ and so on. Now if we require that this Lagrangian be invariant under the gauge transformations $\delta \Psi_{\mu \nu}=\partial_{\mu} \xi_{\nu}-\partial_{\nu} \xi_{\mu}$ with unconstrained spin-vector parameter $\xi_{\mu}$, we get:

$$
\partial^{\mu} \frac{\delta \mathcal{L}_{0}}{\delta \bar{\Psi}^{\mu \nu}}=0 \quad \Rightarrow \quad a_{1}=-2, \quad a_{2}=2, \quad a_{3}=-1, \quad a_{4}=-\frac{1}{2}
$$

Again a first signal that something happens in $d=4$ comes from the contraction with $\gamma$-matrix:

$$
\gamma^{\mu} \frac{\delta \mathcal{L}_{0}}{\delta \bar{\Psi}^{\mu \nu}}=\frac{i(d-4)}{2}\left[-2(\partial \Psi)_{\nu}+2 \hat{\partial}(\gamma \Psi)_{\nu}+2 \gamma_{\nu}(\partial \gamma \Psi)+\partial_{\nu}(\gamma \gamma \Psi)-\gamma_{\nu} \hat{\partial}(\gamma \gamma \Psi)\right]
$$

Noting that in $d=4$ the last term in the Lagrangian containing five $\gamma$-s is not independent, we proceed rewriting the Lagrangian in terms of completely antisymmetric products. Using the relation:

$$
\begin{aligned}
\Gamma^{\mu \nu} \gamma^{\alpha} \Gamma^{\beta \gamma}= & -\left(g^{\mu \beta} g^{\nu \gamma}-g^{\mu \gamma} g^{\nu \beta}\right) \gamma^{\alpha}+\left(-g^{\mu \alpha} g^{\nu \beta} \gamma^{\gamma}+g^{\mu \alpha} g^{\nu \gamma} \gamma^{\beta}+g^{\nu \alpha} g^{\mu \beta} \gamma^{\gamma}-g^{\nu \alpha} g^{\mu \gamma} \gamma^{\beta}\right)+ \\
& +\left(g^{\nu \gamma} g^{\alpha \beta} \gamma^{\mu}-g^{\mu \gamma} g^{\alpha \beta} \gamma^{\nu}-g^{\nu \beta} g^{\alpha \gamma} \gamma^{\mu}+g^{\mu \beta} g^{\alpha \gamma} \gamma^{\nu}\right)+\left(g^{\nu \alpha} \Gamma^{\mu \beta \gamma}-g^{\mu \alpha} \Gamma^{\nu \beta \gamma}\right)+ \\
& +\left(g^{\alpha \beta} \Gamma^{\mu \nu \gamma}-g^{\alpha \gamma} \Gamma^{\mu \nu \beta}\right)+\left(g^{\mu \beta} \Gamma^{\nu \alpha \gamma}-g^{\nu \beta} \Gamma^{\mu \alpha \gamma}-g^{\mu \gamma} \Gamma^{\nu \alpha \beta}+g^{\nu \gamma} \Gamma^{\mu \alpha \beta}\right)+\Gamma^{\mu \nu \alpha \beta \gamma}
\end{aligned}
$$


we obtain for the most general Lagrangian:

$$
\begin{aligned}
\frac{1}{i} \mathcal{L}_{0}= & \left(1-a_{2}-2 a_{4}\right) \bar{\Psi}^{\mu \nu} \hat{\partial} \Psi_{\mu \nu}+\left(a_{1}+a_{2}-2 a_{3}+4 a_{4}\right)\left((\bar{\Psi} \partial)^{\mu}(\gamma \Psi)_{\mu}+(\bar{\Psi} \gamma)^{\mu}(\partial \Psi)_{\mu}\right)+ \\
& +\left(a_{2}+4 a_{4}\right) \bar{\Psi}_{\mu \nu} \Gamma^{\nu \alpha \beta} \Psi_{\mu \beta}+\left(a_{3}-2 a_{4}\right)\left((\bar{\Psi} \partial)_{\mu} \Gamma^{\mu \nu \alpha} \Psi_{\nu \alpha}-\bar{\Psi}_{\mu \nu} \Gamma^{\mu \nu \alpha}(\partial \Psi)_{\alpha}\right)+ \\
& +a_{4} \bar{\Psi}_{\mu \nu} \Gamma^{\mu \nu \alpha \beta \gamma} \partial_{\alpha} \Psi_{\beta \gamma}= \\
= & -\frac{1}{2} \bar{\Psi}_{\mu \nu} \Gamma^{\mu \nu \alpha \beta \gamma} \partial_{\alpha} \Psi_{\beta \gamma}
\end{aligned}
$$

where the last line corresponds to gauge invariant case. Again in this form the gauge invariance of the Lagrangian is evident as well as the fact that it is identically zero in $d=4$.

The last form of the Lagrangian is very simple and convenient. In particular, it makes deformation into $A d S$ space a simple task. Indeed, let us consider the Lagrangian:

$$
\mathcal{L}_{0}=\frac{i}{2} \bar{\Psi}_{\mu \nu} \Gamma^{\mu \nu \alpha \beta \gamma} D_{\alpha} \Psi_{\beta \gamma}
$$

where ordinary partial derivative is replaced by $A d S$ covariant one. Note that the Lagrangian is completely antisymmetric in all vector indices, so that covariant derivative effectively acts on the spinor index only, e.g.:

$$
\left[D_{\mu}, D_{\nu}\right] \xi_{\alpha}=-\frac{\kappa}{2} \Gamma_{\mu \nu} \xi_{\alpha}, \quad \kappa=\frac{2 \Lambda}{(d-1)(d-2)}
$$

Due to non-commutativity of covariant derivatives this Lagrangian is not invariant under the covariantized gauge transformations $\delta \Psi_{\mu \nu}=D_{\mu} \xi_{\nu}-D_{\nu} \xi_{\mu}$ any more:

$$
\delta_{0} \mathcal{L}_{0}=\frac{i \kappa}{4}(d-3)(d-4) \bar{\Psi}_{\mu \nu} \Gamma^{\mu \nu \alpha} \xi_{\alpha}
$$

To compensate this non-invariance we add mass-like term to the Lagrangian as well as corresponding correction to gauge transformations:

$$
\mathcal{L}_{1}=\frac{M}{2} \bar{\Psi}_{\mu \nu} \Gamma^{\mu \nu \alpha \beta} \Psi_{\alpha \beta}, \quad \delta_{1} \Psi_{\mu \nu}=i \alpha_{0}\left(\gamma_{\mu} \xi_{\nu}-\gamma_{\nu} \xi_{\mu}\right)
$$

Simple calculations show that the gauge invariance of the Lagrangian can indeed be restored provided:

$$
\alpha_{0}=-\frac{M}{(d-4)}, \quad M^{2}=-\frac{(d-4)^{2}}{4} \kappa
$$

where the last relation clearly shows that the procedure works in $A d S$ space $(\kappa<0)$ only. Thus we obtain the following Lagrangian and gauge transformations:

$$
\begin{aligned}
\mathcal{L}_{0} & =\frac{i}{2} \bar{\Psi}_{\mu \nu} \Gamma^{\mu \nu \alpha \beta \gamma} D_{\alpha} \Psi_{\beta \gamma}+\frac{M}{2} \bar{\Psi}_{\mu \nu} \Gamma^{\mu \nu \alpha \beta} \Psi_{\alpha \beta} \\
\delta \Psi_{\mu \nu} & =D_{\mu} \xi_{\nu}-D_{\nu} \xi_{\mu}-\frac{i M}{(d-4)}\left(\gamma_{\mu} \xi_{\nu}-\gamma_{\nu} \xi_{\mu}\right)
\end{aligned}
$$

describing massless antisymmetric second rank tensor in $A d S_{d}$ space with $d \geq 5$. 


\section{Massive case}

In this Section we consider gauge invariant description of massive antisymmetric second rank tensor in arbitrary $(A) d S_{d}$ space with $d \geq 5$. Spin-tensor $\Psi_{\mu \nu}$ has gauge transformations with spin-vector parameter, so for gauge invariant description of massive tensor we have introduce additional Goldstone field $\Phi_{\mu}$. This field has its own gauge transformations with spinor parameter, but due to reducibility of gauge transformations for spin-tensor $\Psi_{\mu \nu}$ it is not necessary to introduce any other additional fields. We have already seen that completely antisymmetric products of $\gamma$ matrices plays an essential role, so we will look for the Lagrangian in the form:

$$
\begin{aligned}
\mathcal{L}= & \frac{i}{2} \bar{\Psi}_{\mu \nu} \Gamma^{\mu \nu \alpha \beta \gamma} D_{\alpha} \Psi_{\beta \gamma}+i \bar{\Phi}_{\mu} \Gamma^{\mu \nu \alpha} D_{\nu} \Phi_{\alpha}+ \\
& +\frac{M}{2} \bar{\Psi}_{\mu \nu} \Gamma^{\mu \nu \alpha \beta} \Psi_{\alpha \beta}+i a_{1}\left(\bar{\Psi}_{\mu \nu} \Gamma^{\mu \nu \alpha} \Phi_{\alpha}+\bar{\Phi}_{\mu} \Gamma^{\mu \nu \alpha} \Psi_{\nu \alpha}\right)+a_{2} \bar{\Phi}_{\mu} \Gamma^{\mu \nu} \Phi_{\nu}
\end{aligned}
$$

At the same time we will use the following ansatz for the gauge transformations:

$$
\begin{aligned}
\delta \Psi_{\mu \nu} & =D_{\mu} \xi_{\nu}-D_{\nu} \xi_{\mu}+i \alpha_{0}\left(\gamma_{\mu} \xi_{\nu}-\gamma_{\nu} \xi_{\mu}\right)+\alpha_{1} \Gamma_{\mu \nu} \eta \\
\delta \Phi_{\mu} & =D_{\mu} \eta+\alpha_{2} \xi_{\mu}+i \alpha_{3} \gamma_{\mu} \eta
\end{aligned}
$$

First of all we calculate all variations with one derivative and require their cancellation. It allows us to express all parameters in gauge transformations in terms of the ones in the Lagrangian:

$$
\alpha_{0}=-\frac{M}{(d-4)}, \quad \alpha_{1}=\frac{2 a_{1}}{(d-3)(d-4)}, \quad \alpha_{2}=-2 a_{1}, \quad \alpha_{3}=\frac{a_{2}}{d-2}
$$

Now we calculate all variations without derivatives taking into account contributions of kinetic terms due to non-commutativity of covariant derivatives. Their cancellation gives:

$$
M^{2}=\frac{2(d-4)}{(d-3)} a_{1}^{2}-\frac{(d-4)^{2}}{16} \kappa, \quad a_{2}=-\frac{(d-2)}{(d-4)} M
$$

Working with gauge invariant description of massive fields it is natural to define massless limit as a one where (all) Goldstone field(s) decouple from the main gauge one. For the case at hands we see that it is the limit $a_{1} \rightarrow 0$ that corresponds to the massless one. As for the concrete mass normalization, we will use convention "mass is what would be mass in flat Minkowski space". Using the following relation:

$$
\bar{\Psi}^{\mu \nu} \Psi_{\mu \nu}-2(\bar{\Psi} \gamma)^{\mu}(\gamma \Psi)_{\mu}-\frac{1}{2}(\bar{\Psi} \gamma \gamma)(\gamma \gamma \Psi)=-\frac{1}{2} \bar{\Psi}_{\mu \nu} \Gamma^{\mu \nu \alpha \beta} \Psi_{\alpha \beta}
$$

we obtain:

$$
a_{1}^{2}=\frac{(d-3)}{2(d-4)} m^{2}, \quad M^{2}=m^{2}-\frac{(d-4)^{2}}{4} \kappa
$$

Collecting all results together we obtain the following Lagrangian:

$$
\begin{aligned}
\mathcal{L}= & \frac{i}{2} \bar{\Psi}_{\mu \nu} \Gamma^{\mu \nu \alpha \beta \gamma} D_{\alpha} \Psi_{\beta \gamma}+i \bar{\Phi}_{\mu} \Gamma^{\mu \nu \alpha} D_{\nu} \Phi_{\alpha}+\frac{M}{2} \bar{\Psi}_{\mu \nu} \Gamma^{\mu \nu \alpha \beta} \Psi_{\alpha \beta}+ \\
& +i \sqrt{\frac{(d-3)}{2(d-4)}} m\left(\bar{\Psi}_{\mu \nu} \Gamma^{\mu \nu \alpha} \Phi_{\alpha}+\bar{\Phi}_{\mu} \Gamma^{\mu \nu \alpha} \Psi_{\nu \alpha}\right)-\frac{(d-2)}{(d-4)} M \bar{\Phi}_{\mu} \Gamma^{\mu \nu} \Phi_{\nu}
\end{aligned}
$$


invariant under the following gauge transformations:

$$
\begin{aligned}
\delta \Psi_{\mu \nu} & =D_{\mu} \xi_{\nu}-D_{\nu} \xi_{\mu}-\frac{2 i M}{(d-4)}\left(\gamma_{\mu} \xi_{\nu}-\gamma_{\nu} \xi_{\mu}\right)+\sqrt{\frac{2}{(d-3)(d-4)}} \frac{m}{(d-4)} \Gamma_{\mu \nu} \eta \\
\delta \Phi_{\mu} & =D_{\mu} \eta-\sqrt{\frac{2(d-3)}{(d-4)}} m \xi_{\mu}-i \frac{M}{(d-4)} \gamma_{\mu} \eta
\end{aligned}
$$

where $M^{2}=m^{2}-\frac{(d-4)^{2}}{4} \kappa$. Note that this time our construction works in $d S$ space $(\kappa>0)$ as well but for $m^{2} \geq \frac{(d-4)^{2}}{4} \kappa$ only. At the boundary of this region (as it is often to be the case for fermionic fields) the Lagrangian and gauge transformations become much simpler:

$$
\begin{aligned}
\mathcal{L}= & \frac{i}{2} \bar{\Psi}_{\mu \nu} \Gamma^{\mu \nu \alpha \beta \gamma} D_{\alpha} \Psi_{\beta \gamma}+i \bar{\Phi}_{\mu} \Gamma^{\mu \nu \alpha} D_{\nu} \Phi_{\alpha}+ \\
& +i \sqrt{\frac{(d-3)}{2(d-4)}} m\left(\bar{\Psi}_{\mu \nu} \Gamma^{\mu \nu \alpha} \Phi_{\alpha}+\bar{\Phi}_{\mu} \Gamma^{\mu \nu \alpha} \Psi_{\nu \alpha}\right) \\
\delta \Psi_{\mu \nu}= & D_{\mu} \xi_{\nu}-D_{\nu} \xi_{\mu}+\sqrt{\frac{2}{(d-3)(d-4)}} \frac{m}{(d-4)} \Gamma_{\mu \nu} \eta \\
\delta \Phi_{\mu}= & D_{\mu} \eta-\sqrt{\frac{2(d-3)}{(d-4)}} m \xi_{\mu}
\end{aligned}
$$

\section{Arbitrary rank}

In this Section we consider generalization of the above results to the case of completely antisymmetric spin-tensors of arbitrary rank. The higher the rank the more and more complicated become the calculations, thus we will not consider the most general possibilities here. Instead, simply assuming that completely antisymmetric products of $\gamma$ matrices do the job, we will try to construct gauge invariant description of massive spin-tensor of rank $n$ in $(A) d S_{d}$ space with $d \geq 2 n+1$. Again due to reducibility of gauge transformations we will need two spin-tensors with ranks $n$ and $n-1$ only: $\Psi_{\mu_{1} \ldots \mu_{n}}$ and $\Phi_{\mu_{1} \ldots \mu_{n-1}}$. By analogy with $n=2$ case we will look for the Lagrangian in the following form:

$$
\begin{aligned}
\mathcal{L}= & \frac{i}{n !} \bar{\Psi}_{\mu_{1} \ldots \mu_{n}} \Gamma^{\mu_{1} \ldots \mu_{n} \alpha \nu_{1} \ldots \nu_{n}} D_{\alpha} \Psi_{\nu_{1} \ldots \nu_{n}}+\frac{i}{(n-1) !} \bar{\Phi}_{\mu_{1} \ldots \mu_{n-1}} \Gamma^{\mu_{1} \ldots \mu_{n-1} \alpha \nu_{1} \ldots \nu_{n-1}} D_{\alpha} \Phi_{\nu_{1} \ldots \nu_{n-1}}+ \\
& +\frac{M}{n !} \bar{\Psi}_{\mu_{1} \ldots \mu_{n}} \Gamma^{\mu_{1} \ldots \mu_{n} \nu_{1} \ldots \nu_{n}} \Psi_{\nu_{1} \ldots \nu_{n}}+\frac{a_{2}}{(n-1) !} \bar{\Phi}_{\mu_{1} \ldots \mu_{n-1}} \Gamma^{\mu_{1} \ldots \mu_{n-1} \nu_{1} \ldots \nu_{n-1}} \Phi_{\nu_{1} \ldots \nu_{n-1}}+ \\
& +\frac{i a_{1}}{(n-1) !}\left(\bar{\Psi}_{\mu_{1} \ldots \mu_{n}} \Gamma^{\mu_{1} \ldots \mu_{n} \nu_{1} \ldots \nu_{n-1}} \Phi_{\nu_{1} \ldots \nu_{n-1}}+\bar{\Phi}_{\mu_{1} \ldots \mu_{n-1}} \Gamma^{\mu_{1} \ldots \mu_{n-1} \nu_{1} \ldots \nu_{n}} \Psi_{\nu_{1} \ldots \nu_{n}}\right)
\end{aligned}
$$

Similarly, we introduce the following ansatz for gauge transformations:

$$
\begin{aligned}
\delta \Psi_{\mu_{1} \ldots \mu_{n}} & =D_{\left[\mu_{1}\right.} \xi_{\left.\mu_{2} \ldots \mu_{n}\right]}+i \alpha_{0} \gamma_{\left[\mu_{1}\right.} \xi_{\left.\mu_{2} \ldots \mu_{n}\right]}+\alpha_{1} \Gamma_{\left[\mu_{1} \mu_{2}\right.} \eta_{\left.\mu_{3} \ldots \mu_{n}\right]} \\
\delta \Phi_{\mu_{1} \ldots \mu_{n-1}} & =D_{\left[\mu_{1}\right.} \eta_{\left.\mu_{2} \ldots \mu_{n-1}\right]}+\alpha_{2} \xi_{\mu_{1} \ldots \mu_{n-1}}+i \alpha_{3} \gamma_{\left[\mu_{1}\right.} \eta_{\left.\mu_{2} \ldots \mu_{n-1}\right]}
\end{aligned}
$$


where brackets denote antisymmetrization with weight one. Again we begin with the calculation of all variations with one derivative and require their cancellation. It allows us to express all parameters in gauge transformations in terms of the one in the Lagrangian:

$$
\begin{gathered}
\alpha_{0}=(-1)^{n-1} \frac{M}{(d-2 n)}, \quad \alpha_{1}=\frac{2 a_{1}}{(d-2 n)(d-2 n+1)} \\
\alpha_{2}=-n a_{1}, \quad \alpha_{3}=(-1)^{n} \frac{a_{2}}{(d-2 n+2)}
\end{gathered}
$$

Then we calculate all variations without derivatives taking into account contributions of kinetic terms due to non-commutativity of covariant derivatives. And indeed all of them cancel provided:

$$
M^{2}=\frac{n(d-2 n)}{(d-2 n+1)} a_{1}^{2}-\frac{(d-2 n)^{2}}{4} \kappa, \quad a_{2}=-\frac{(d-2 n+2)}{(d-2 n)} M
$$

As in the previous case, we see that it is the limit $a_{1} \rightarrow 0$ corresponds to the massless limit. In this, our convention on mass normalization gives:

$$
a_{1}^{2}=\frac{(d-2 n+1)}{n(d-2 n)} m^{2}, \quad M^{2}=m^{2}-\frac{(d-2 n)^{2}}{4} \kappa
$$

Again the whole construction works in $d S$ space $(\kappa>0)$ as well but for $m^{2} \geq \frac{(d-2 n)^{2}}{4} \kappa$ only.

\section{Conclusion}

Thus really there is no contradiction between new and previous results on antisymmetric second rank spin-tensor. The resolution turns out to be simple (though may be unexpected): the gauge invariant Lagrangian does exists in $d \geq 5$, but in $d=4$ it becomes zero thus explaining why all previous attempts were unsuccessful. The simple completely antisymmetric on all vector indices form of the Lagrangian obtained, turns out to be very convenient in practical calculations such as deformation into $A d S$ space or introduction of mass. Thus it seems worthwhile to reconsider the problem of possible interactions for such fields.

\section{References}

[1] P. K. Townsend "Gauge Invariance for Spin 1/2", Phys. Lett. B90 (1980) 275.

[2] S. Deser, E. Witten "Dynamical Properties of Antisyymetric Tensor Fields", Nucl. Phys. B178 (1980) 491.

[3] S. Deser, P. K. Townsend, W. Siegel "Higher Rank Representations of Lower Spin", Nucl. Phys. B184 (1981) 333.

[4] J. Niederle, A. G. Nikitin "Relativistic wave equations for interacting massive particles with arbitrary half-intreger spins", Phys. Rev. D64 (2001) 125013, arXiv:hep-th/0412213. 
[5] I. L. Buchbinder, V. A. Krykhtin, L. L. Ryskina "Lagrangian formulation of massive fermionic totally antisymmetric tensor field theory in $A d S_{d}$ space", arXiv:0902.1471.

[6] I. L. Buchbinder, V. A. Krykhtin "Gauge invariant Lagrangian construction for massive bosonic higher spin fields in D dimensions", Nucl. Phys. B727 (2005) 537, arXiv:hep-th/0505092.

[7] I. L. Buchbinder, V. A. Krykhtin, L .L. Ryskina, H. Takata "Gauge invariant Lagrangian construction for massive higher spin fermionic fields", Phys. Lett. B641 (2006) 386, arXiv:hep-th/0603212.

[8] I. L. Buchbinder, V. A. Krykhtin, P. M. Lavrov "Gauge invariant Lagrangian formulation of higher spin massive bosonic field theory in AdS space", Nucl. Phys. B762 (2007) 344, arXiv:hep-th/0608005.

[9] I. L. Buchbinder, V. A. Krykhtin, A. A. Reshetnyak "BRST approach to Lagrangian construction for fermionic higher spin fields in (A)dS space", Nucl. Phys. B787 (2007) 211, arXiv:hep-th/0703049.

[10] P. Yu. Moshin, A. A. Reshetnyak "BRST approach to Lagrangian formulation for mixedsymmetry fermionic higher-spin fields", JHEP 10 (2007) 040, arXiv:0707.0386.

[11] I. L. Buchbinder, V. A. Krykhtin, H. Takata "Gauge invariant Lagrangian construction for massive bosonic mixed symmetry higher spin fields", Phys. Lett. B656 (2007) 253, arXiv:0707.2181.

[12] I. L. Buchbinder, V. A. Krykhtin, L. L. Ryskina "BRST approach to Lagrangian formulation of bosonic totally antisymmeric tensor fields in curved space", arXiv:0810.3467.

[13] S. M. Klishevich, Yu. M. Zinoviev "On electromagnetic interaction of massive spin-2 particle", Phys. Atom. Nucl. 61 (1998) 1527, arXiv:hep-th/9708150.

[14] Yu. M. Zinoviev "On Massive High Spin Particles in (A)dS", arXiv:hep-th/0108192.

[15] R. R. Metsaev "Gauge invariant formulation of massive totally symmetric fermionic fields in (A)dS space", Phys. Lett. B643 (2006) 205-212, arXiv:hep-th/0609029.

[16] Yu. M. Zinoviev "Frame-like gauge invariant formulation for massive high spin particles", Nucl. Phys. B808 (2009) 185, arXiv:0808.1778.

[17] Yu. M. Zinoviev "Towards frame-like gauge invariant formulation for massive mixed symmetry bosonic fields", Nucl. Phys. B812 (2009) 46, arXiv:0809.3287.

[18] I. L. Buchbinder, A. V. Galajinsky "Quartet unconstrained formulation for massive higher spin fields", JHEP 0811 (2008) 081, arXiv:0810.2852. 